\title{
Immersive high fidelity simulation of critically ill patients to study cognitive errors: a pilot study
}

\author{
Shivesh Prakash ${ }^{1,2^{*}}$, Shailesh Bihari ${ }^{2}$, Penelope Need ${ }^{3}$, Cyle Sprick ${ }^{4}$ and Lambert Schuwirth ${ }^{1,5}$
}

\begin{abstract}
Background: The majority of human errors in healthcare originate from cognitive errors or biases. There is dearth of evidence around relative prevalence and significance of various cognitive errors amongst doctors in their first post-graduate year. This study was conducted with the objective of using high fidelity clinical simulation as a tool to study the relative occurrence of selected cognitive errors amongst doctors in their first post-graduate year.

Methods: Intern simulation sessions on acute clinical problems, conducted in year 2014, were reviewed by two independent assessors with expertise in critical care. The occurrence of cognitive errors was identified using Likert scale based questionnaire and think-aloud technique. Teamwork and leadership skills were assessed using Ottawa Global Rating Scale.

Results: The most prevalent cognitive errors included search satisfying (90\%), followed by premature closure (PC) (78.6\%), and anchoring (75.7\%). The odds of occurrence of various cognitive errors did not change with time during internship, in contrast to teamwork and leadership skills $\left(x^{2}=11.9, P=0.01\right)$. Anchoring appeared to be significantly associated with delay in diagnoses $(P=0.007)$ and occurrence of PC $(P=0.005)$. There was a negative association between occurrence of confirmation bias and the ability to make correct diagnosis $(P=0.05)$.

Conclusions: Our study demonstrated a high prevalence of anchoring, premature closure, and search satisfying amongst doctors in their first post-graduate year, using high fidelity simulation as a tool. The occurrence of selected cognitive errors impaired clinical performance and their prevalence did not change with time.
\end{abstract}

Keywords: Cognitive bias, Decision-making, Diagnostic errors, Patient simulation, Physicians

\section{Background}

There has been increasing impetus worldwide on preventing medical errors, which have been estimated to be one of the leading contributors to poor outcome including mortality [1]. An important type of medical error concern diagnosis and clinical decision making, since they are highly prevalent, often preventable, and generally lead to greater morbidity and mortality than other types of error [2]. In fact, the majority of complaints brought against emergency physicians arise from delayed or missed diagnoses [3]. These errors in diagnosis and clinical decision

\footnotetext{
*Correspondence: shivesh.prakash@health.sa.gov.au

'Prideaux Centre for Research in Health Professions Education, Flinders

University, Bedford Park, South Australia 5042, Australia

${ }^{2}$ Department of Intensive care, Flinders Medical Centre, 1 Flinders drive,

Bedford Park, South Australia 5042, Australia

Full list of author information is available at the end of the article
}

making have been shown to arise due to technical/ knowledge deficits, system related factors or cognitive errors. Of these cognitive errors have been shown to be major contributors (in more than three fourths of errors involving diagnosis and clinical decision making) $[4,5]$.

Cognitive errors may stem from inappropriate application or complete failure to apply knowledge or suboptimal 'cognitive dispositions to respond' (CDR) or biases $[4,6,7]$. The term CDR has been suggested to replace the term 'bias', to remove the negative connotations associated with the later [8]. These biases represent the manner in which a physician orientates and responds to the presenting complaints, symptoms and signs under circumstances of uncertainty [8]. Cognitive biases can prompt clinicians to make errors when pruning, selecting and/or validating a diagnosis resulting in missed/wrong 
diagnoses and treatment [6, 9-15]. There are several factors which predispose to cognitive errors such as fatigue and sleep deprivation, affective state, patient factors, ambient conditions and past experiences [8].

The cognitive errors have been studied extensively in high-stakes safety industry such as aviation [16, 17]. However, in the medical literature the focus has been relatively recent $[6,18-22]$. Also, the evidence on cognitive errors largely originates from studies on speciality trainees, and reveal that practicing physicians and trainees demonstrate considerable susceptibility to various cognitive errors. The initial post graduate years involve a sharp learning curve [23], wherein foundations of their non-technical and technical skills are laid with implications on their future performance. Therefore, identifying the common cognitive errors and how they impact performance can help prioritising them for focused interventions [24, 25]. In the absence of a formal training in the current curriculum, clinical experience alone would have to be relied on to attenuate the harmful effects of cognitive errors. However, whether this is the case, and to what extent is unknown.

To study cognitive errors in medicine, doctors are usually made to engage in cognitive tasks of diagnosis synthesis and decision making under experimental conditions. The majority of studies have used case notes and lowfidelity computer screen-based simulations to engage doctors in these cognitive tasks. The degree of immersion, cognitive, physical and affective load imposed by widely used methods of case notes and computer screen-based simulations, is different from what doctors experience in real life setting $[26,27]$. Although there is controversy as to the learning efficacy of low versus high-fidelity simulation; [28] for research studies on cognitive errors, the latter may be better suited [27, 29-31]. Hence, clinical problems, recreated in a high-fidelity simulated environment, with engineered complexity, uncertainty and temporal demands, is likely to reflect the real life challenges more closely. Observing and interviewing doctors who immerse and engage with patients and other staff in such an environment provides an opportunity for assessment of various technical and non-technical skills including diagnosis synthesis and decision making [32]. Occurrence of various cognitive errors and their impact on diagnosis synthesis and decision making can also be studied [25, 32], as various factors such as content knowledge, scenario complexity, environment, time of the day, experience etc. can be controlled to certain degree.

In this pilot study, we investigate the relative occurrence of selected cognitive errors amongst doctors in their first post-graduate year, using high fidelity clinical simulation as a tool.

Besides cognitive errors, the changes in teamwork and leadership skills were also assessed during the first post graduate year; as this is one of the main learning objectives of the existing simulation program.

\section{Methods}

The study involved review of intern (PGY1) simulation sessions conducted in year 2014 at Flinders medical centre, South Australia. The study was approved by Southern Adelaide Clinical Human Research Ethics Committee (91.15).

\section{Simulation program}

Simulation involved high-fidelity scenarios, using the SimMan simulator (Laerdal Medical, Wappingers Falls, NY, USA), and actors (trained simulation centre staff) representing other team members (surgeon, nurse, medical emergency registrar etc.). Each session started with briefing, followed by simulation and a debriefing session. Video assisted debriefing [CAE LearningSpace ${ }^{\mathrm{m} x}$ (CAE Healthcare, Canada)] was used with focus on technical skills, teamwork and leadership. The predominant debriefing style involved advocacy inquiry [33]. This involves pairing observations of participant's actions (or inaction) with inquiry into participants' thought processes during that moment.

The four simulation scenarios chosen for study were-(A) acute onset breathlessness in a post-operative knee surgery patient, secondary to cardiogenic pulmonary edema; (B) pain in abdomen and dizziness in a day 1 post hemicolectomy patient who has deteriorating hypovolemic shock (covert to overt), secondary to intra-abdominal bleeding; (C) low-conscious state in a trauma patient secondary to narcotic overdose resulting from a prescription error, and (D) low-conscious state due to hypoglycaemia in a fasting diabetic patient awaiting surgery, where regular insulin dose was not withheld (Appendix). These scenarios were developed from actual incidents in hospital for learning purposes. In each quarter of internship year, the interns were exposed to one of the four scenarios. The sequence of exposure to these scenarios over the year was random. Each simulation scenario began with the nurse calling the doctor to see the unwell patient. Towards the end of session, the senior help (usually an ICU or admitting consultant) enters and requests a handover from the team.

Think-aloud: In think-aloud studies, people are asked to verbalize their thinking while performing tasks [34]. Researchers using this technique typically both observe and audiotape or videotape the participant [35]. Although it is often used, the technique is not entirely undisputed and there is some criticism in literature that thinking aloud and the limited capacity of memory may hinder the cognitive processes of the participant, thus affecting performance [36-38]. On the other hand, there is broad support for it as well, and it is often seen as the only available 
'next best thing' to-the currently impossible-'direct observation' of thought processes [39]. An alteration to the conventional 'think-aloud' protocol is the use of 'verbal probing techniques' [40]. Immediately following a particular response, questions (probes), are asked to help reveal the thought process [41]. Investigators have previously found that asking post-process questions to subjects provided valuable information that made think aloud data easier to understand and interpret $[42,43]$.

The participants in our study were not given instructions beforehand to verbalize their thoughts. Instead their thought process was probed (prompts) by the patient, the nurse and the 'senior help' to help reveal their thoughts during simulation. Prompts by the patient were initiated when the participant appeared to have gathered initial patient data i.e. history and physical exam. Prompts such as "what's wrong with me doctor?" and "what does that mean?" were used to get insight into working differentials and participant's understanding of pathophysiology and synthesis from the clinical data. The prompts from the nurse involved "what are you thinking?" and "why do you think so?". These prompts were initiated if the participant initiated treatments without verbalizing the diagnosis or clinical problem or the participant verbalizes the diagnosis without the basis for it. The processing framework for think-aloud data was similar to that described previously [44]. Verbal data was transcribed and broken down into smaller components by predefined codes such as signs, symptoms, action, treatment, labs, monitoring. Scripts were then analysed to ascertain relationship between codes and identification of inductive and deductive reasoning processes.

Towards the end of session, the senior help arrived and asked the participants to verbalize their assessment of the situation and actions, along with the basis for it. If the participants were not correct with the diagnosis, they were asked if there could be other differentials and what were the findings for and against each differential. By comparing responses against checklist of key expectations, this interview process allowed for gaining an estimate about their knowledge around pertinent diagnoses and management. For example, the participant was identified to have adequate knowledge if he/she was aware of bradypnoea, hypothermia and pin-point pupils, and use of naloxone as a part of narcotic toxodrome diagnosis and management respectively.

\section{Recording of cognitive errors}

For pragmatic reasons, only selected cognitive errors (Table 1) were studied, of the more than 30 reported in literature [8]. The basis for selecting these was the prevalence as reported in previous studies [45], minimal overlap in theoretical construct, coverage of both diagnosis synthesis and treatment decisions and feasibility of
Table 1 Catalogue of cognitive errors used in this study [45]

\begin{tabular}{|c|c|}
\hline 1. Anchoring & $\begin{array}{l}\text { Tendency to fixate on a specific feature } \\
\text { of a presentation early in the diagnostic } \\
\text { process at the expense of understanding } \\
\text { whole situation. }\end{array}$ \\
\hline 2. Confirmation Bias & $\begin{array}{l}\text { Seeking or acknowledging only information } \\
\text { that confirms the desired or suspected } \\
\text { diagnosis. As new information is available } \\
\text { there is a tendency to select out information } \\
\text { which supports initial hypothesis, rather } \\
\text { than adjusting the initial hypothesis in the } \\
\text { light of new information against the } \\
\text { initial hypothesis. }\end{array}$ \\
\hline 3. Premature Closure & $\begin{array}{l}\text { Accepting a diagnosis prematurely, failure } \\
\text { to consider reasonable differential of } \\
\text { possibilities. There is an obvious halt to } \\
\text { the diagnostic process targeted } \\
\text { at differentials. }\end{array}$ \\
\hline 4. Search satisfying & $\begin{array}{l}\text { Once a diagnosis is made, there may be } \\
\text { a tendency to stop searching for co-existing } \\
\text { diagnoses or causes and complications of } \\
\text { current diagnosis. }\end{array}$ \\
\hline 5. Commission bias & $\begin{array}{l}\text { Tendency toward action rather than inaction. } \\
\text { Performing un-indicated manoeuvres, } \\
\text { deviating from protocol. May be due to } \\
\text { overconfidence, desperation, or pressure } \\
\text { from others. }\end{array}$ \\
\hline 6. Omission bias & $\begin{array}{l}\text { Hesitation to start emergency manoeuvres } \\
\text { for fear of being wrong or causing harm, } \\
\text { tendency towards inaction. }\end{array}$ \\
\hline 7. Overconfidence & $\begin{array}{l}\text { Tendency to act on incomplete information, } \\
\text { intuitions or hunches. Too much faith is } \\
\text { placed in opinion instead of carefully } \\
\text { gathered evidence. Often reluctant accept } \\
\text { suggestions to consider alternatives. }\end{array}$ \\
\hline
\end{tabular}

studying them based on video review of simulation sessions. The simulation scenarios were developed to focus on technical skills (diagnosis and treatment) and nontechnical skills of teamwork and leadership.

To identify the occurrence of cognitive errors, two investigators ( $\mathrm{SP}$ and $\mathrm{SB}$ ) independently analysed the video recording and 'think aloud' comments from participants [CAE LearningSpace ${ }^{\mathrm{Tm}}$ (CAE Healthcare, Canada)]. The observations were recorded using a Likert scale based questionnaire tool (Table 2). This questionnaire was adapted from a previously published questionnaire [45], which was used in a similar context of simulated inhospital acutely sick patient. The assessors, similar to our study, were trained and had expertise in the field. Also, the questionnaire was demonstrated to be reliable (Cronbach's $\alpha$ 0.81). Both the investigators have expertise in the management of critically ill patients and underwent training at using this questionnaire, which involved (i) familiarising themselves with the theoretical constructs of cognitive errors and the questionnaire, (ii) going through published examples of such cognitive errors, (iii) observing 10 randomly selected simulation sessions and applying questionnaire and discussing 
Table 2 Questionnaire tool to assess cognitive errors

\begin{tabular}{|c|c|}
\hline 1 & $\begin{array}{l}\text { Initiated critical treatments in a timely manner and at } \\
\text { appropriate dose or extent }\end{array}$ \\
\hline 2 & Does not allocate attention to all presenting issues \\
\hline 3 & $\begin{array}{l}\text { Treatments initiated which were not indicated based on } \\
\text { established emergency procedures (desperation, "try anything") }\end{array}$ \\
\hline 4 & $\begin{array}{l}\text { Requires prompting to call for help/resources or refuses to } \\
\text { call upon prompting }\end{array}$ \\
\hline 5 & Did not consider a thorough differential diagnosis \\
\hline 6 & Tried to "make" new data/information fit a diagnosis \\
\hline 7 & $\begin{array}{l}\text { Once diagnosis is made searches for all causes of diagnosis. } \\
\text { For eg. Does not stop searching for other fentanyl patches } \\
\text { when one patch found. }\end{array}$ \\
\hline 8 & Recognition of problem is promptly followed by intervention \\
\hline 9 & $\begin{array}{l}\text { Became focused on one issue at the expense of fully } \\
\text { understanding the situation }\end{array}$ \\
\hline 10 & $\begin{array}{l}\text { Treatments initiated or actions undertaken which were not } \\
\text { necessary }\end{array}$ \\
\hline 11 & $\begin{array}{l}\text { Initiated treatments at inappropriate dose without } \\
\text { checking/confirming/consulting }\end{array}$ \\
\hline 12 & $\begin{array}{l}\text { Tendency to anchor on to salient feature early in the } \\
\text { diagnosis process }\end{array}$ \\
\hline 13 & $\begin{array}{l}\text { Did not alter the diagnosis when hints were provided by } \\
\text { nurse or patient to alternative diagnosis }\end{array}$ \\
\hline 14 & $\begin{array}{l}\text { Once diagnosis is made searches for all complications of } \\
\text { the diagnosis }\end{array}$ \\
\hline
\end{tabular}

differences if any and (iv) discussing how in each of the four study scenarios, the specific cognitive error may manifest. While both the investigators were aware of the main study objective of feasibility, one investigator (SB) was blinded to other research questions. The investigators indicated whether or not a particular cognitive error was made by a given participant using a five point Likert scale, with the following anchors: 'Strongly disagree'; 'Disagree'; 'Neutral/Unsure'; 'Agree'; 'Strongly agree'. The theoretical construct of cognitive errors obtained from previous publication allowed for two items per error, without increasing the risk of redundancy, cognitive load and fatigue on the assessors, while still preserving the face validity. Anchoring was reflected by questions 2 and 9, commission by questions 3 and 10, confirmation by questions 6 and 13, overconfidence by questions 4 and 11 , omission by questions 1 and 8 , premature closure by questions 5 and 12, and search satisfying by questions 7 and 14. For each cognitive error, the scores were averaged to develop final score that reflected three final outcomes-'Present', 'absent' and 'can't be assessed or unsure'. A cognitive error was considered as either present or absent, if both the assessors agreed. The outcome was 'unsure/can't be assessed' if marked by both the assessors as 'unsure/can't be assessed' or there was difference in marking.

\section{Recording of non-technical skills}

The non-technical skills were evaluated using previously validated, Ottawa Crisis Resource Management Global Rating Scale (Ottawa GRS) [46, 47]. The Ottawa GRS is divided into five categories of crisis resource management (CRM) skills based on recognized CRM literature: problem solving, situational awareness, leadership, resource utilization and communication. An overall rating category for CRM performance is also provided. Each category is measured on a seven-point anchored ordinal scale with descriptive anchors to provide guidelines on alternating points along the scale.

\section{Statistical methods}

Both descriptive and inferential statistics were used. The results are presented using tables and graphs. Data is presented as median and Interquartile range (IQR), unless otherwise specified. Prevalence of cognitive errors is reported as a ratio of number of observed error type to total number of scenarios. Normality was assessed using Kolmogorov-Smirnov test. Associations between categorical and continuous variables were assessed using Chisquare test and Spearman's Rho respectively. The relationship of delay in diagnosis and time spent in internship, with the occurrence of cognitive error, was studied after adjusting for scenario type, using generalized linear modelling and logistic regression. Time to diagnosis (dependent variable) across scenario types (factor variable) was compared using one way ANOVA. Trends in Ottawa GRS score over the PGY1 year was assessed using the Friedman test. The internal consistency and inter-rater agreement (for the final outcomes of 'Present,' 'absent' and 'can't be assessed or unsure') of questionnaire tool for assessment of cognitive errors was analysed using Cronbach's alpha and Kappa statistic respectively. The thresholds for Cronbach's alpha [48] and Kappa statistic [49] were 0.7 and 0.6 respectively. Analysis was performed using SPSS version 21 (IBM Corp., Armonk, NY, USA). For all tests, a two-sided $P$ value of less than 0.05 was considered significant.

\section{Results}

A total of 70 simulation sessions were reviewed over the year 2014. A total of 25 interns participated in these sessions as primary responder. There were 17 sessions each of cardiogenic pulmonary oedema, narcotic overdose and hypoglycaemia, and 19 sessions of haemorrhagic shock.

\section{Performance of questionnaire}

The inter-rater agreement and internal consistency was good for all cognitive errors assessed, except 'overconfidence' (Cronbach's alpha 0.46; Kappa statistic 0.17 for overconfidence). 


\section{Overall prevalence of cognitive errors}

Total number of studied cognitive errors observed per session ranged from 0 to 5 , with a median (IQR) of 3 (2-4) errors per session. Frequent cognitive errors, observed in more than $75 \%$ of sessions, included search satisfying (90\%), followed by premature closure (78.6\%), and anchoring (75.7\%) (Fig. 1). No instances of overconfidence were identified. The uncertainty was most frequent in the assessment of omission bias; whereas it was least frequent in the assessment of anchoring and search satisfying.

\section{Impact of occurrence of cognitive errors on diagnosis synthesis}

The correct diagnosis was reached in 57 (85.1\%) sessions, with a median (IQR) time of 4.05 (2.3-6) minutes. The scenarios differed in time to reach diagnosis $(P=0.04)$. After adjusting for the scenario type, anchoring appeared to be significantly associated with delay in diagnoses $(\beta=0.2,95 \%$ CI 0.5 to $3.0, P=0.007)$ and also occurrence of premature closure $\left(x^{2}=9.4, P=0.005\right)$. There was a trend towards negative association between occurrence of confirmation bias and the ability to make a correct diagnosis $\left(\mathrm{x}^{2}=5.8, P=0.05\right)$.

\section{Occurrence of cognitive errors with time spent in internship}

As shown in Table 3, there was no association of occurrence of particular cognitive error with scenario type, except for omission bias. After adjusting for scenario type, the odds of occurrence of various cognitive errors did not change with time spent in the internship year (Table 3).

\section{Other non-technical skills}

There was overall improvement of OTTAWA GRS scores during the internship period $\left(x^{2}=11.9, P=0.01\right)$ (Fig. 2). As shown in Fig. 2, there was improvement in skills involving leadership $\left(x^{2}=28, P<0.001\right)$, problem solving $\left(x^{2}=8.1\right.$, $P=0.04)$, resource utilization $\left(\mathrm{x}^{2}=19.7, P<0.001\right)$ and communication skills $\left(\mathrm{x}^{2}=19.4, p<0.001\right)$. No significant trend was seen in situational awareness $\left(\mathrm{x}^{2}=1.9, P=0.6\right)$.

Review of interview between 'senior help' and the participant revealed that although all participants had knowledge of clinical presentation of these diagnoses/syndromes, there were frequent deficits in the knowledge around management.

\section{Discussion}

Our study shows that cognitive errors are highly prevalent amongst junior doctors in their first post-graduate year, particularly anchoring, premature closure and search satisfying. Although junior doctors' clinical experience increased during the year leading to a significant improvement of their non-technical skills of teamwork and leadership, there was no change in the occurrence of studied cognitive errors. This is unfortunate because we also found that anchoring and confirmation bias did not only impact on the ability to make a diagnosis, but also resulted in a delay in making a diagnosis. Moreover, anchoring was associated with premature closure.

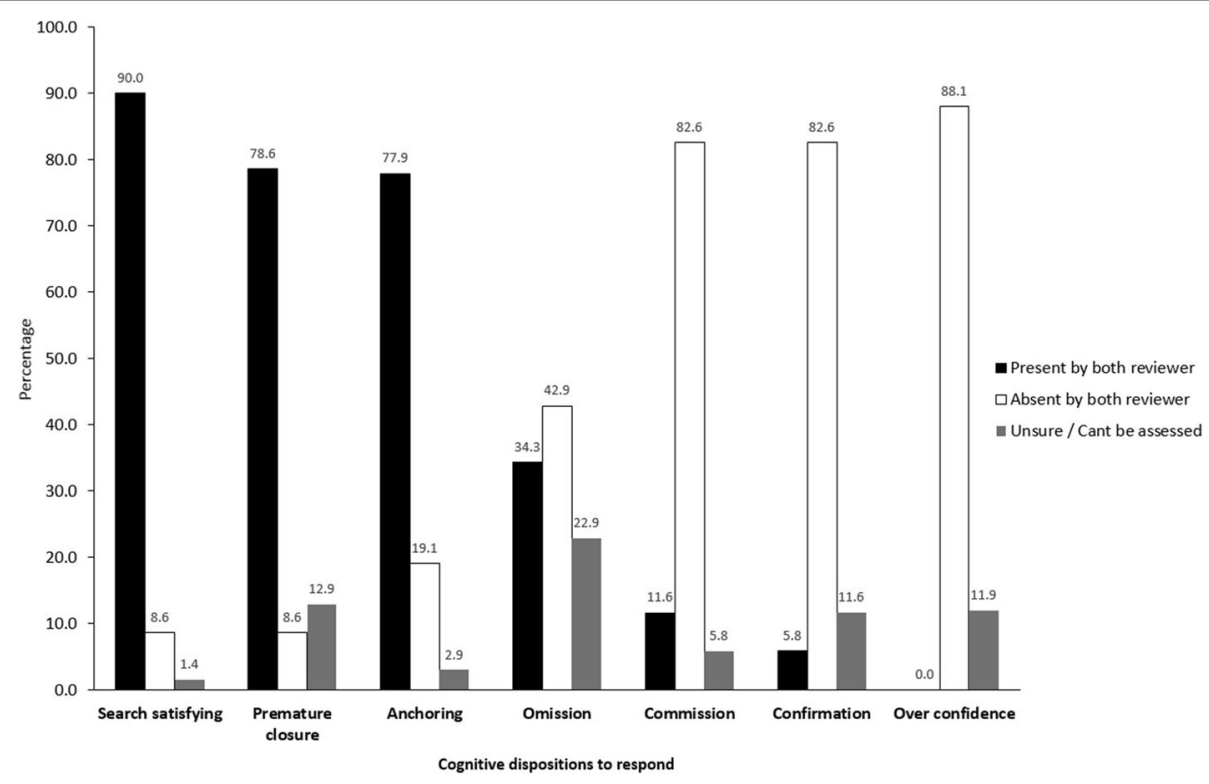

Fig. 1 Overall prevalence of cognitive errors 
Table 3 Association of cognitive errors with scenario types and odds of their occurrence with increasing internship time exposure

\begin{tabular}{|c|c|c|c|c|}
\hline \multirow[t]{2}{*}{ Cognitive error } & \multicolumn{2}{|c|}{ Association with scenario type } & \multicolumn{2}{|c|}{ Odds of occurrence with increasing internship time exposure } \\
\hline & $\overline{x^{2}}$ & $P$ value & $\mathrm{OR}(95 \% \mathrm{Cl})$ & $P$ value \\
\hline Search satisfying & 0.47 & 0.9 & $1.2(0.89 ; 1.7)$ & 0.21 \\
\hline Premature closure & 2.0 & 0.57 & $0.75(0.52 ; 1.08)$ & 0.12 \\
\hline Anchoring & 2.4 & 0.4 & $0.84(0.66 ; 1.07)$ & 0.15 \\
\hline Commission & 3.4 & 0.3 & $0.99(0.75 ; 1.33)$ & 0.91 \\
\hline Confirmation bias & 2.2 & 0.52 & $1.02(0.68 ; 1.55)$ & 0.91 \\
\hline Omission bias & 10.3 & 0.02 & $1.3(0.94 ; 1.9)$ & 0.11 \\
\hline
\end{tabular}

Observed cognitive errors and their impact on diagnosis synthesis

The most frequent cognitive errors were 'anchoring', 'premature closure' and 'search satisfying'. Anchoring was significantly associated with delay in reaching a correct diagnosis and premature closure. Anchoring represents the tendency to fixate on the hypothesis generated from a specific feature of a presentation early in the diagnostic process, at the expense of understanding the whole situation. Recovery from this initial anchor

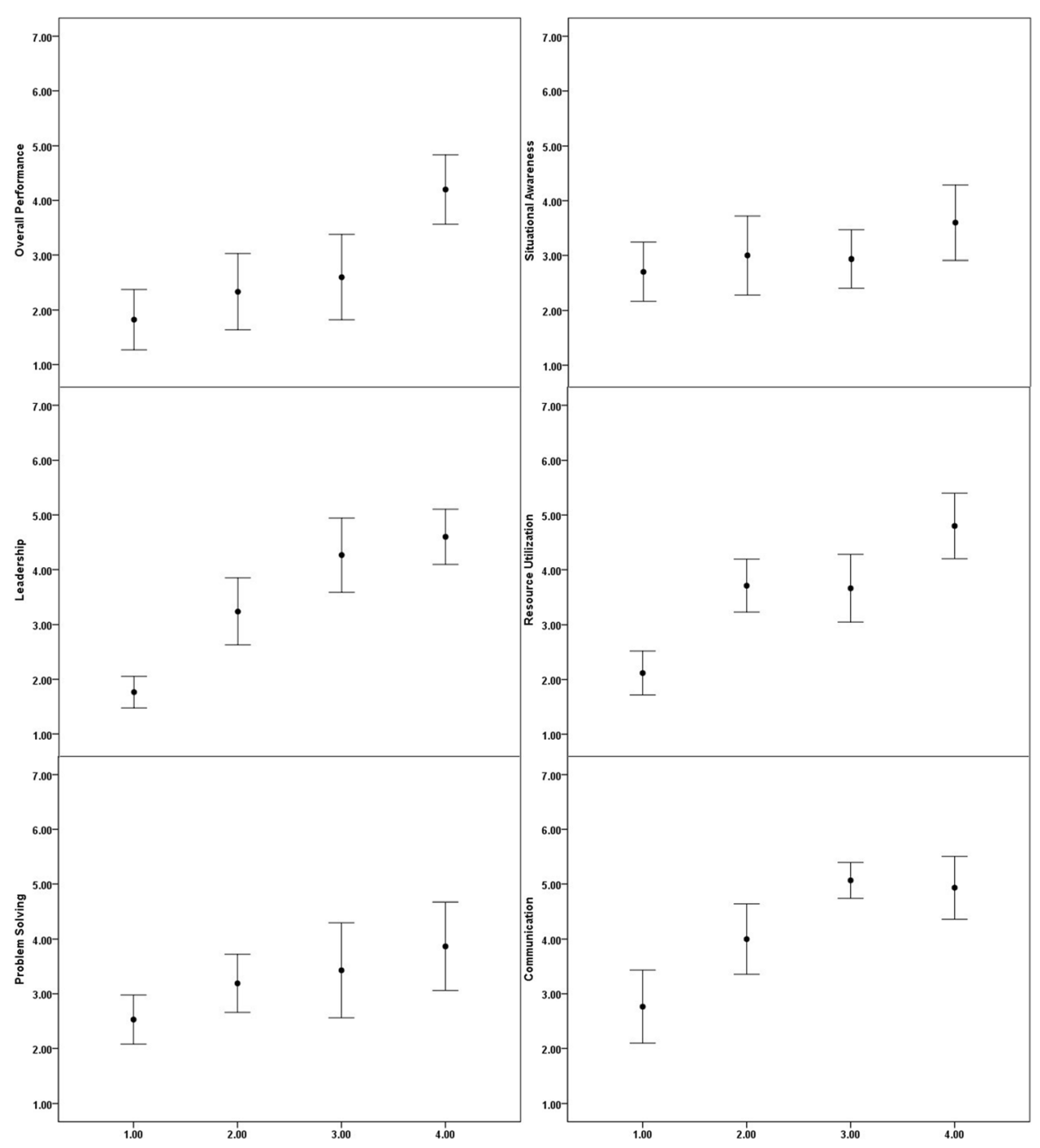

Fig. 2 The Ottawa GRS score for non-technical skills across four quarters during the internship year 
involves 'adjusting' to more appropriate diagnosis, by seeking additional data for and against the diagnostic hypothesis generated from the initial feature [50]. This is therefore likely to delay reaching correct diagnosis by the adjusting process [50], either rendered insufficient by premature closure, or flawed by confirmation bias. In confirmation bias, time is spent to recruit selective information to reinforce the initial impression that might have been generated due to anchoring; in which case, the correct diagnosis may not be even considered. The occurrence of confirmation bias becomes more likely when the information is presented sequentially, as is usually the case in clinical medicine [51]. This also reinforces the value of high-fidelity patient simulation for studying cognitive errors over case notes or computer screen-based tools, where the information is available upfront. The highly prevalent search satisfying by definition resulted in high incidence of failure to search for causes or contributing factors, complications and co-existing diagnoses. Attempts at identifying these are likely to result in more successful and effective management.

Overconfidence bias was not observed in our study. This is contrary to findings in the literature of considerable prevalence [12]. However, susceptibility to overconfidence bias may vary with seniority and may actually be low in fresh medical graduates, as supported by findings of Friedman et al. [52].

Despite the high prevalence of cognitive errors, the correct diagnosis was considered in majority of sessions. This is perhaps not as illogical as it seems: heuristics and biases that may underlie these errors, evolve over time to help perform under circumstances of uncertainty and high cognitive load, and hence they may not be always associated with poor outcome [53]. Also some of the cognitive errors studied may not impact diagnostic accuracy, but may influence the choices in management, eg. search satisfying may lead to missed co-diagnoses or complications. Increase in complexity of case, temporal demand or fatigue may uncover the potentially harmful effects of biases or cognitive dispositions. Besides these possibilities, it has also been shown that even if the correct diagnosis appears as one of the differentials, it is rarely rejected during the diagnostic process [54].

\section{High fidelity simulation as a research and teaching tool}

Our pilot study is one of the first that demonstrates the feasibility of immersive high-fidelity clinical simulations to study cognitive errors, combined with a think aloud technique and a questionnaire based tool. This is a step forwards from previous studies utilizing predominantly case notes or computer screen based tools to study cognitive errors. Our study demonstrated the utility of simulation in eliciting how they may negatively impact clinical performance such as diagnosis synthesis and treatment decisions. Guided reflection here is likely to help develop catalogue of examples of situations where the potentially harmful effects of biases or cognitive dispositions may occur (exemplar theory [55]) and also motivate participants to learn (motivation theory [56]).

There was a significant improvement in teamwork and leadership skills. The efficacy of simulation based training for these skills have been demonstrated extensively in literature [57]. Similarly, focused simulation based training could help doctors to recognize and manage situations where they are at risk for cognitive errors. The applied psychology concepts, common to simulation based learning, of metacognition and 'de-biasing' strategies have been demonstrated in other fields to be effective in cognitive error prevention and recovery [58-61].

In the absence of formal training, clinical experience is often relied on to mitigate cognitive errors. In our study, 1 year of clinical experience did not appear to influence the occurrence of various cognitive errors. It is unlikely that the context of scenarios alone would have yielded this result as: (i) the sequence of scenarios were not fixed, (ii) the pattern of occurrence of cognitive errors across scenarios was largely similar and (iii) we adjusted for scenario type in the regression analysis. Since the study was not designed for this research question, the finding can only be regarded as hypothesis generating, to be tested in a larger prospective study. In support of this finding, however, is a prospective observation study which revealed that the clinical reasoning process remains relatively constant from medical school entry to practice [62]. Also, the high prevalence of cognitive errors amongst speciality trainees and practicing physicians suggests that experienced clinicians are as likely as junior colleagues to experience adverse cognitive dispositions or biases [63, 64]. There could be few reasons as to why clinical experience alone may not suffice as a remedy for cognitive errors. Firstly, the outcomes in real life may not be immediately visible and secondly, the learning that occurs from experience require unbiased and informed reflection (Kolb's experiential theory) [65] on what cognitive errors are and how they can impair clinical performance. Observing clinicians during their work to enhance experiential learning around cognitive errors has ethical limitations as the error would need to be interrupted or ideally avoided, so that the patient is not harmed. Here simulation based learning may be beneficial as the errors can be allowed to evolve and serve as a learning catalyst.

\section{Resources involved in delivering high fidelity simulation based teaching}

Simulation based teaching intervention can be resource intensive, and there is limited data in literature. Lapkin et al. reported that marginal cost of high fidelity simulation 
amounted to \$ 291.26 per participant [66]. For our simulation based teaching, we used the resource cost model to estimate the cost [67]. There is one time cost of setting up of simulation facility which also includes purchase and installation of Mannequin, simulation software and computers. There are also recurring costs, of which the significant ones include equipment and materials, and personnel cost. Under our business model, the simulation based activity in the current project was a part of a larger simulation and clinical skills programme, used for training of medical students, post graduate doctors, nurses and allied staff. Hence the above costs which otherwise are significant (approximately $\$ 200,000$ ) would be distributed across several teaching programs. Similarly, the cost involved in scenario development and programming was about $\$ 364.62$ per scenario $(6 \mathrm{~h}-\$ 60.08 / \mathrm{h})$. This cost again would be distributed across 30 to 40 participants each year, thereby significantly reducing the cost per participant. Per participant, the personnel cost involved in simulation based teaching was $\$ 157$ (0.5 h nursing time, $0.6 \mathrm{~h}$ of 2 medical facilitators and $0.3 \mathrm{~h}$ technical and administrative time).

\section{Limitations and future research}

We used retrospective study design which allowed for assessment of authentic performance of participants and their thought process using think-aloud transcripts. Although we were able to control for uncertainty, a prospective study using direct interview may help minimize uncertainty in assessment and also further validate the questionnaire based tool. The inter-rater agreement and internal consistency in assessment of Overconfidence was poor. This is likely to be highly skewed observations towards absence of Overconfidence.

Although, the assessors were well trained for making observations, there is no independent corroboration of assessor's observations. Also only one of the two assessors was blinded to the research questions. Although this may have not affected the main objective of the study, it may impact on specific hypothesis testing. In future studies, it is essential that the assessors are blinded to the hypothesis being tested.

We used modified think-aloud technique using verbal probes. Although such technique allows for more focused information gathering, while minimizing interruptions, it can potentially introduce bias. Certain probe questions can be perceived as a cue to reconsider their thoughts/decisions. Despite their usefulness, unfortunately both conventional think-aloud technique and verbal probing have inherent shortcomings which are difficult to overcome [36-38]. Besides think aloud technique, there are other methods such as script concordance approach [68] and post-hoc cognitive walkthroughs [69] using video review, which may be trialled in future studies.
Cognitive errors were detected based on outcome measures, which could have been influenced by factors such as knowledge deficit. Since our assessment revealed deficits in knowledge around management of the simulated syndromes/diagnoses, some of these encountered errors may have originated from lack of knowledge, such as omission bias. This highlights the importance of assessment of the relevant content knowledge of participants, in order to better assess the origin of observed cognitive error. This is important to know, as interventions would differ. We did not have pre-defined standardized framework for assessment. Hence, although there was flexibility to modify assessment to the context, scenario and participant; this resulted in heterogeneity in assessment technique. A pre-formulated structured assessment framework will allow uniformity in assessment and needs to be trialled in a prospective study.

The results of our study need to be triangulated with a larger prospective study on cognitive errors using immersive high fidelity simulation as a tool. Also by controlling for various factors such as complexity of scenario, cognitive load, participant experience etc., and more objective assessment of participant knowledge base, it needs to be determined as to how and under what circumstances the cognitive dispositions or biases result in negative outcome. This is likely to provide essential evidence to facilitate studies into how simulation based education could be used to train doctors to avoid, identify and recover from cognitive errors.

\section{Conclusions}

Our study demonstrated a high prevalence of anchoring, premature closure, and search satisfying amongst doctors in their 1st postgraduate year. The clinical experience gained during their first post graduate year and simulation based training focused on teamwork and leadership was associated with acquisition of these skills. However, there was no change in the prevalence of various cognitive errors. Further prospective research is needed to validate the results and explore the utility of simulation based intervention to help doctors manage their cognitive errors to improve clinical reasoning.

\section{Appendix}

Details of scenarios with examples of cognitive errors.

\section{Scenario A: cardiogenic pulmonary edema}

Clinical problem: Acute onset breathlessness in a postoperative knee surgery patient. Participant expected to manage acutely sick patient and work through differentials of sudden onset breathlessness.

Story and setting: The doctor is summoned by the bedside nurse to assess an elderly patient who is 3 days post knee replacement. He is in an orthopaedic ward, 
and develops sudden onset shortness of breath at rest. He has significant hypoxia and tachypnoea, which poorly responds to high oxygen delivery via non-rebreather mask. He is anxious, diaphoretic and cyanosed. He does not tolerate lying down. On auscultation the participant finds bilateral wheeze, basal crepts on lung auscultation and gallop rhythm on cardiac auscultation. He has cool peripheries but with strong pulse. He is in fast atrial fibrillation and is significantly hypertensive (BP systolic $210 \mathrm{mmHg}$ ). There is no calf swelling/fever and operative site appears unremarkable, except for tenderness. He does not have chest pain but complains of tightness and difficulty breathing. He is not a diabetic, but does have a history of ischemic heart disease. He is one DVT prophylaxis and anti-platelet agents. During assessment, the patient at one instance coughs up pink frothy secretions.

Examples of cognitive traps:

\begin{tabular}{|c|c|}
\hline CDR & Scenario specific examples \\
\hline Anchoring & $\begin{array}{l}\text { The participant displays a tendency to fixate } \\
\text { on ECG changes/possibility of DVT/Bilateral } \\
\text { wheezing and possibility of acute asthma } \\
\text { early on in their workup. }\end{array}$ \\
\hline Premature closure & $\begin{array}{l}\text { Given the history of knee surgery, the participants } \\
\text { commonly display premature closure with diagnosis } \\
\text { of pulmonary embolism. }\end{array}$ \\
\hline Search satisfying & $\begin{array}{l}\text { Once recognizing the pattern of cardiogenic } \\
\text { pulmonary edema, the participants either } \\
\text { stop searching for likely causes or stop } \\
\text { beyond the likelihood of ischemic cardiac event. }\end{array}$ \\
\hline Confirmation & $\begin{array}{l}\text { When thinking of pulmonary embolism, } \\
\text { they display tendency to interpret post-operative } \\
\text { knee pain as a feature of DVT and pink } \\
\text { frothy secretions as 'haemoptysis' of pulmonary } \\
\text { embolism. They then display tendency to } \\
\text { ignore features against, such as orthopnoea, } \\
\text { hypertension, and auscultation findings. }\end{array}$ \\
\hline Overconfidence & $\begin{array}{l}\text { Ignoring suggestion for calling for help by } \\
\text { the nurse, despite deteriorating condition. } \\
\text { Making guesses on medication dosage, } \\
\text { despite available option of checking. }\end{array}$ \\
\hline Commission & $\begin{array}{l}\text { On deterioration, tendency to lie the patient } \\
\text { down, despite marked orthopnoea. Tendency } \\
\text { to commence IV fluid/administer fluid bolus, } \\
\text { despite hypertension. }\end{array}$ \\
\hline Omission & $\begin{array}{l}\text { Despite, marked hypoxia, commencing } \\
\text { oxygen delivery at very low rate } \\
\text { (eg } 2-4 \mathrm{~L} / \text { min nasal specks) and then } \\
\text { tendency to not escalate it further. } \\
\text { Despite acknowledging very high blood pressure, } \\
\text { not commencing treatment interventions. }\end{array}$ \\
\hline
\end{tabular}

Scenario B: hypovolemic shock secondary to bleeding Clinical problem: Pain in abdomen and dizziness in a day 1 post hemi-colectomy patient who has deteriorating hypovolemic shock (covert to overt) secondary to intraabdominal bleeding.
Story and setting: The doctor is summoned by the bedside nurse to assess an elderly patient who is day 1 , post hemi-colectomy and is complaining of abdominal pain poorly responding to ordered analgesics and dizziness. The patient is awake, anxious and diaphoretic. She complains of pain in the upper abdomen which is dull and poorly localized. The abdomen seems to be distended. The patient does have surgical drain which has poured out significant blood in the past $2 \mathrm{~h}$, and also the patient has urinary catheter with poor urine output, but this information needs to be actively sought by the assessor (As the nurse is unaware of this). The patient's blood pressure is normal initially but has sinus tachycardia, with cold peripheries and poor capillary refill. If the haemorrhagic shock is not recognized and managed appropriately, the patient's condition deteriorates with narrowing of pulse pressure, followed by overt hypotension. In a completely neglected case, pulseless electrical activity ensues. If blood tests done, reveals low haemoglobin.

Examples of cognitive traps:

\begin{tabular}{|c|c|}
\hline$\overline{C D R}$ & Scenario specific examples \\
\hline Anchoring & $\begin{array}{l}\text { The participants often anchor onto control of pain as } \\
\text { the main issue. Also upper abdominal pain results in } \\
\text { anchor into acute coronary syndrome. Some } \\
\text { participants anchor onto abdominal distention with } \\
\text { concerns of bowel distention and resort to placement } \\
\text { of naso-gastric tube as a priority. All these examples } \\
\text { involve failure to fully assess, gather all information } \\
\text { and synthesizing the bigger picture. Another example } \\
\text { would be recognizing low blood pressure and fixating } \\
\text { on it using repeated fluid challenges, rather than } \\
\text { working through the cause and treating it. }\end{array}$ \\
\hline
\end{tabular}

Premature Post-operative ileus, myocardial infarction and closure analgesic management are examples of diagnoses, associated with failure to think through other possibilities.

Search satisfying Once the haemorrhagic shock was recognized, there was failure to search for cause and complications. Example, efforts to look for anti-platelet agents or anti-coagulation, renal failure, hypothermia, medications causing low BP etc.

Confirmation An example would be actively seeking abdominal $\mathrm{x}$-ray and placement of nasogastric tube for abdominal distention, despite recognizing evolving shock and bleeding from the surgical drain.

Overconfidence Ignoring suggestion for calling for help by the nurse, despite deteriorating condition. Making guesses on medication dosage, despite available option of checking.

Commission Upon deteriorating using bag mask ventilation, despite patient breathing by herself. Some even elected to use GTN with the premature closure around myocardial infarction, despite the evolving shock.

Omission Not commencing fluid bolus, or not giving fluid bolus beyond the initial bolus, or hesitancy in commencing bolus and only commencing slow infusion despite marked hypotension. Another example is not asking for blood transfusion despite noticing blood in the post-surgical drain. 


\section{Scenario C: narcotic overdose}

Clinical problem: low-conscious state in a trauma patient secondary to narcotic overdose resulting from prescription error.

Story and setting: The doctor is summoned by the nurse to assess this patient who is difficult to arouse. The patient was admitted last evening following a fall. He has bruise on his head and has fracture of his right wrist. He is due operation for his broken wrist later in the day. Last evening had a head CT which was normal (this information is available, if the participant asks for). He has two fentanyl patches which he applied himself before coming to hospital. Also there is duplication of opioid prescription (this information has to be actively sought by the participant, as the nurse is unaware). The patient has signs of opioid overdose-pinpoint pupils, low respiratory rate, low heart rate, and low body temperature. The participant is expected to work through causes of low conscious state, detect opioid toxodrome and treat accordingly.

Examples of cognitive traps:

\begin{tabular}{|c|c|}
\hline CDR & Scenario specific examples \\
\hline Anchoring & $\begin{array}{l}\text { The participant had a tendency to anchor } \\
\text { on the bruise and fixate on possibility of } \\
\text { head injury. Other examples include anchoring } \\
\text { on low sinus heart rate and focusing on } \\
\text { workup for causes of sinus bradycardia, rather } \\
\text { than looking at the bigger picture and } \\
\text { synthesising the probability of narcotic } \\
\text { overdose. }\end{array}$ \\
\hline Premature closure & $\begin{array}{l}\text { Premature closure was often seen around } \\
\text { the diagnosis of head injury. }\end{array}$ \\
\hline Search satisfying & $\begin{array}{l}\text { Once opioid toxodrome was recognized, } \\
\text { efforts were not made to identify the cause } \\
\text { such as the additional fentanyl patch and } \\
\text { duplication of opioid order. Other causes } \\
\text { were not looked for/excluded such as } \\
\text { electrolyte imbalance, hypoglycaemia other } \\
\text { drug overdose. }\end{array}$ \\
\hline Confirmation & $\begin{array}{l}\text { Asking for another head } C T \text {, looking at the } \\
\text { bruise. This was despite negative head } \\
\text { CT } 12 \mathrm{~h} \text { back. }\end{array}$ \\
\hline Overconfidence & $\begin{array}{l}\text { Ignoring suggestion for calling for help by } \\
\text { the nurse, despite deteriorating condition. } \\
\text { Making guesses on medication dosage, } \\
\text { despite available option of checking. }\end{array}$ \\
\hline Commission & $\begin{array}{l}\text { Upon deteriorating using bag mask } \\
\text { ventilation, despite patient breathing by } \\
\text { herself. Using atropine to increase heart rate. } \\
\text { Using fluid bolus despite normal blood } \\
\text { pressure. }\end{array}$ \\
\hline Omission & $\begin{array}{l}\text { Not using naloxone or hesitancy to re-administer } \\
\text { or commence naloxone infusion. }\end{array}$ \\
\hline
\end{tabular}

\section{Scenario D: hypoglycaemia}

Clinical problem: low-conscious state due to hypoglycaemia in a fasting diabetic patient awaiting surgery, where regular insulin dose was not withheld.

Story and setting: The doctor is summoned by the nurse to assess this elderly patient who is confused and more somnolent. The patient was admitted last evening following a knife injury to her hand. Overnight, she has been having pain issues, needing analgesics (including opioids). The patient is due tendon repair operation this morning and has been fasting for the same. Despite fasting, the patient got given insulin overnight (this info was not known to nurse and has to be sought by the participant). The patient is severely hypoglycaemic. The participant was expected to work through causes of low conscious state, detect hypoglycaemia and it's cause and treat accordingly.

Examples of cognitive traps:

\begin{tabular}{|c|c|}
\hline CDR & Scenario specific examples \\
\hline Anchoring & $\begin{array}{l}\text { The participant had a tendency to fixate on } \\
\text { confusion in elderly in the setting of pain. } \\
\text { Some had a tendency to fixate on opioid use } \\
\text { and some on the nature of wound injury and } \\
\text { possibility of sepsis. }\end{array}$ \\
\hline Premature closure & $\begin{array}{l}\text { Premature closure was often seen around the } \\
\text { clinical syndromes of delirium, dementia, } \\
\text { opioid toxodrome }\end{array}$ \\
\hline Search satisfying & $\begin{array}{l}\text { Once hypoglycaemia was detected, efforts } \\
\text { were not made to find out the cause } \\
\text { (prescription error) and hence prevention of } \\
\text { further hypoglycaemia. }\end{array}$ \\
\hline Confirmation & $\begin{array}{l}\text { Despite no response to naloxone or no history } \\
\text { of dementia and in a } 12 \mathrm{~h} \text { clean wound, } \\
\text { persistent efforts to explore opioid, psychiatric } \\
\text { and sepsis hypothesis }\end{array}$ \\
\hline Overconfidence & $\begin{array}{l}\text { Ignoring suggestion for calling for help by } \\
\text { the nurse, despite deteriorating condition. }\end{array}$ \\
\hline Commission & $\begin{array}{l}\text { Upon deteriorating using bag mask ventilation, } \\
\text { despite patient breathing by herself. Using fluid } \\
\text { bolus despite normal blood pressure. }\end{array}$ \\
\hline Omission & $\begin{array}{l}\text { Despite noticing long acting insulin } \\
\text { prescription, failure to hold that prescription } \\
\text { and commencement of dextrose infusion to } \\
\text { prevent recurrence. }\end{array}$ \\
\hline
\end{tabular}

Abbreviations

CRM: Crisis resource management; GRS: Global rating scale; IQR: Interquartile range

\section{Acknowledgments}

We would like to acknowledge the support of simulation lab staff at Flinders University in providing administrative assistance in the conduct of this study. We would also like to acknowledge the logistical support and infrastructure provided by Dr Christine Burdeniuk, Director of Clinical Training at Flinders Medical centre for the conduct of this study. 


\section{Funding}

The project was a part of paid research fellowship of the first author.

\section{Availability of data and materials}

All study instruments used are included within the article and as supplement. The raw dataset supporting the conclusions of this article is available from the first author upon request.

\section{Authors' contributions}

SP was involved in study design conception, data gathering and analysis and manuscript preparation. SB was involved in data gathering and statistical analysis. PN and CS were involved in refining of methodology and manuscript preparation. LS was involved in study design conception, supervision, analysis, critical review and substantial contribution in discussion of study results in light of current literature. All authors read and approved the final manuscript.

\section{Competing interests}

The authors declare that they have no competing interests.

\section{Consent for publication}

Not applicable as all participants in the study are anonymous.

\section{Ethics approval and consent to participate}

The study was approved by Southern Adelaide Clinical Human Research Ethics Committee (91.15). The requirement for consent was waived.

\section{Author details}

${ }^{1}$ Prideaux Centre for Research in Health Professions Education, Flinders University, Bedford Park, South Australia 5042, Australia. ${ }^{2}$ Department of Intensive care, Flinders Medical Centre, 1 Flinders drive, Bedford Park, South Australia 5042, Australia. ${ }^{3}$ Director of General Practice Training, Flinders Medical Centre, Flinders Drive, Bedford Park, SA 5042, Australia. ${ }^{4}$ Simulation Unit, School of Medicine - Flinders University, Bedford Park, South Australia 5042, Australia. ${ }^{5}$ Health Professions Education, School of Medicine, Flinders University, Bedford Park, South Australia 5042, Australia.

\section{Received: 30 September 2016 Accepted: 26 January 2017}

\section{Published online: 08 February 2017}

\section{References}

1. Makary MA, Daniel M. Medical error-the third leading cause of death in the US. BMJ. 2016:353:i2139.

2. Saber Tehrani AS, Lee H, Mathews SC, Shore A, Makary MA, Pronovost PJ, Newman-Toker DE. 25-Year summary of US malpractice claims for diagnostic errors 1986-2010: an analysis from the National Practitioner Data Bank. BMJ Qual Saf. 2013;22:672-80.

3. Data from the U.S. General Accounting Office, the Ohio Hospital Association and the St. Paul (MN) Insurance Company, 1998. http://hookman.com/ mp9807.htm. Accessed 24 Apr 2015.

4. Graber ML, Franklin N, Gordon R. Diagnostic error in internal medicine. Arch Intern Med. 2005;165:1493-9.

5. Harrison BT, Gibberd RW, Hamilton D. An analysis of the causes of adverse events from the Quality in Australian Health Care Study. Med J Aust. 1999; 170(9):411-5.

6. Croskerry P. From Mindless to Mindful Practice-Cognitive Bias and Clinical Decision Making. N Engl J Med. 2013;368:2445-8.

7. Groopman J. How Doctors Think. Boston: Houghton Mifflin; 2008

8. Croskerry P. Diagnostic Failure: A Cognitive and Affective Approach. In: Henriksen K, Battles JB, Marks ES, et al., editors. Advances in Patient Safety: From Research to Implementation (Volume 2: Concepts and Methodology). Rockville: Agency for Healthcare Research and Quality (US); 2005. http:// www.ncbi.nlm.nih.gov/books/NBK20487/. Accessed 24 Apr 2015.

9. Croskerry P. The importance of cognitive errors in diagnosis and strategies to minimize them. Acad Med. 2003:78:775-80.

10. Crowley RS, Legowski E, Medvedeva O, et al. Automated detection of heuristics and biases among pathologists in a computer-based system. Adv Health Sci Educ Theory Pract. 2013;18:343-63.

11. Elstein AS. Heuristics and biases: selected errors in clinical reasoning. Acad Med. 1999;74:791-4.

12. Berner ES, Graber ML. Overconfidence as a cause of diagnostic error in medicine. Am J Med. 2008;121:2-33.
13. Redelmeier $D$. The cognitive psychology of missed diagnoses. Ann Intern Med. 2005;142:115-20.

14. Lee CS, Nagy PG, Weaver SJ, Newman-Toker DE. Cognitive and system factors contributing to diagnostic errors in radiology. AJR Am J Roentgenol. 2013:201:611-7.

15. Croskerry P. Achieving quality in clinical decision making: cognitive strategies and detection of bias. Acad Emerg Med. 2002;9:1184-204.

16. Malakis S, Kontogiannis T, Kirwan B. Managing emergencies and abnormal situations in air traffic control (part II): teamwork strategies. Appl Ergon. 2010;41:628-35.

17. The human factor in nuclear power plant operation. NEA Issue Brief: an analyses of principle nuclear issues. 1998. Report No. 2, January. Website. https://www.oecd-nea.org/brief/brief-02.html. Accessed 24 Apr 2015.

18. Croskerry P, Singhal G, Mamede S. Cognitive debiasing 1: origins of bias and theory of debiasing. BMJ Qual Saf. 2013;22:ii58-64.

19. Ogdie AR, Reilly JB, Pang WG, et al. Seen Through Their Eyes: Residents' Reflections on the Cognitive and Contextual Components of Diagnostic Errors in Medicine. Acad Med. 2012;87:1361-7.

20. Reilly JB, Ogdie AR, Von Feldt JM, Myers JS. Teaching about how doctors think: a longitudinal curriculum in cognitive bias and diagnostic error for residents. BMJ Qual Saf. 2013:22:1044-50.

21. Stiegler MP, Tung A. Cognitive processes in anesthesiology decision making Anesthesiology. 2014;120:204-17.

22. Vickrey BG, Samuels MA, Ropper AH. How neurologists think: a cognitive psychology perspective on missed diagnoses. Ann Neurol. 2010;67:425-33.

23. Hopper AN, Jamison MH, Lewis WG. Learning curves in surgical practice. Postgrad Med J. 2007:83:777-9.

24. Kerfoot BP, DeWolf WC, Masser BA, et al. Spaced education improves the retention of clinical knowledge by medical students: a randomised controlled trial. Med Educ. 2007:41:23-31.

25. Bond WF, Deitrick LM, Eberhardt $M$, et al. Cognitive versus technical debriefing after simulation training. Acad Emerg Med. 2006;13:276-83.

26. Russell JA. A circumplex model of affect. J Pers Soc Psychol. 1980;39:1161-78.

27. Mills BW, Carter OB, Rudd CJ, et al. Effects of Low- Versus High-Fidelity Simulations on the Cognitive Burden and Performance of Entry-Level Paramedicine Students: A Mixed-Methods Comparison Trial Using EyeTracking, Continuous Heart Rate, Difficulty Rating Scales, Video Observation and Interviews. Simul Healthc. 2016:11:10-8.

28. de Giovanni D, Roberts T, Norman G. Relative effectiveness of high- versus low-fidelity simulation in learning heart sounds. Med Educ. 2009:43:661-8.

29. Issenberg SB, McGaghie WC, Petrusa ER, et al. Features and uses of highfidelity medical simulations that lead to effective learning: a BEME systematic review. Med Teach. 2005;27:10-28.

30. Yang $\mathrm{H}$, Thompson $\mathrm{C}$, Bland $\mathrm{M}$. The effect of clinical experience, judgment task difficulty and time pressure on nurses' confidence calibration in a high fidelity clinical simulation. BMC Med Inform Decis Mak. 2012:12:113.

31. Lasater K. High-fidelity simulation and the development of clinical judgment: students' experiences. J Nurs Educ. 2005:46:4.

32. Satish U, Streufert S. Value of a cognitive simulation in medicine: towards optimizing decision making performance of healthcare personnel. Qual Saf Health Care. 2002;11:163-7.

33. Rudolph JW, Simon R, Rivard P, et al. Debriefing with good judgement: Combining rigorous feedback with genuine inquiry. Anesthesiol Clin. 2007; 25:361-76.

34. Newell A, Simmon AH. Human problem solving. Englewood Cliffs: Prentice Hall; 1992

35. Ericsson KA, Simon AH. Protocol analysis: Verbal reports as data. Revth ed. MIT Press: Cambridge; 1993.

36. Kim HS. We talk, therefore we think? A cultural analysis of the effect of talking on thinking. J Pers Soc Psychol. 2002;83:828-42

37. Gilhooly KJ, Fioratou E, Henretty N. Verbalization and problem solving: Insight and spatial factors. Br J Psychol. 2010;101:81-93.

38. Boren T, Ramey J. Thinking aloud: Reconciling theory and practice. IEEE Trans Prof Commun. 2000:43:261-78.

39. Russo JE, Johnson EJ, Stephens DL. The validity of verbal protocols. Mem Cogn. 1989;17:759-69.

40. Willis G, DeMaio T, Harris-Kojetin B. Is the Bandwagon Headed to the Methodological Promised Land? Evaluation of the Validity of Cognitive Interviewing Techniques. In: Sirken M, Herrmann D, Schechter S, Schwarz N, Tanur J, Tourangeau R, editors. Cognition and Survey Research. New York: Wiley; 1999. 
41. Willis GB, Artino AR. What Do Our Respondents Think We're Asking? Using Cognitive Interviewing to Improve Medical Education Surveys. J Grad Med Educ. 2013:5:353-6.

42. Branch JL. Investigating the information-seeking processes of adolescents: The value of using think-alouds and think afters. Libr Inf Sci Res. 2000;22: 371-92.

43. Fonteyn ME, Kuipers B, Grobe SJ. A description of think aloud method and protocol analysis. Qual Health Res. 1993;3:430-41.

44. Lundgrén-Laine $H$, Salanterä S. Think-aloud technique and protocol analysis in clinical decision-making research. Qual Health Res. 2010;20:565-75.

45. Stiegler MP, Neelankavil JP, Canales C, et al. Cognitive errors detected in anaesthesiology: a literature review and pilot study. Br J Anaesth. 2012;108: 229-35.

46. Kim J, Neilipovitz D, Cardinal P, et al. A Comparison of Global Rating Scale and Checklist Scores in the Validation of an Evaluation Tool to Assess Performance in the Resuscitation of Critically III Patients During Simulated Emergencies (Abbreviated as "CRM Simulator Study IB"). Simul Healthc. 2009;4:6-16

47. Kim J, Neilipovitz D, Cardinal P, et al. A pilot study using high-fidelity simulation to formally evaluate performance in the resuscitation of critically ill patients: The University of Ottawa Critical Care Medicine, High-Fidelity Simulation, and Crisis Resource Management I Study. Crit Care Med. 2006; 34:2167-74.

48. Tavakol M, Dennick R. Making sense of Cronbach's alpha. Int J Med Educ. 2011;2:53-5

49. McHugh ML. Interrater reliability: the kappa statistic. Biochem Med (Zagreb). 2012;22:276-82.

50. Epley N, Gilovich T. Putting Adjustment Back in the Anchoring and Adjustment Heuristic: Differential Processing of Self-Generated and Experimenter-Provided Anchors. Psychol Sci. 2001;12:391-6.

51. Jonas E, Schulz-Hardt S, Frey D, et al. Confirmation bias in sequential information search after preliminary decisions: an expansion of dissonance theoretical research on selective exposure to information. J Pers Soc Psychol. 2001;80:557-71.

52. Friedman $C P$, et al. Do physicians know when their diagnoses are correct? Implications for decision support and error reduction. J Gen Intern Med. 2005;20(4):334-9.

53. Todd PM, Gigerenzer G. Précis of Simple heuristics that make us smart. Behav Brain Sci. 2000;23(5):727-41. discussion 742-80.

54. Elstein AS, Shulman LS, Sprafka SA. Medical problem solving: An analysis of clinical reasoning. Cambridge: Harvard University Press; 1978.

55. Doug L, Medin LD, Schaffer MM. Context Theory of Classification Learning. Psychol Rev. 1978;85:207-38.

56. Biehler RF, Snowman J. Psychology applied to teaching. 7th ed. Boston: Houghton Mifflin Company; 1993.

57. Frengley RW, Weller JM, Torrie J, et al. The effect of a simulation-based training intervention on the performance of established critical care unit teams. Crit Care Med. 2011;39:2605-11.

58. Keren G. Cognitive aids and debiasing methods: can cognitive pills cure cognitive ills? In: Caverni JP, Fabre JM, Gonzales M, editors. Cognitive Biases. New York: Elsevier; 1990. p. 523-52.

59. Plous S. The Psychology of Judgment and Decision Making. Philadelphia: Temple University Press; 1993.

60. Fischhoff B. Debiasing. In: Kahneman D, Slovic P, Tversky A, editors. Judgment under Uncertainty: Heuristics and Biases. New York: Cambridge University Press; 1982. p. 422-44.

61. Arkes HA. Impediments to accurate clinical judgment and possible ways to minimize their impact. In: Arkes HR, Hammond KR, editors. Judgment and Decision Making: An Interdisciplinary Reader. New York: Cambridge University Press; 1986. p. 582-92.

62. Neufeld VR, Norman GR, Feightner JW, et al. Clinical problem-solving by medical students: a cross-sectional and longitudinal analysis. Med Educ. 1981;15:315-22

63. Kuhn GJ. Diagnostic errors. Acad Emerg Med. 2002;9:740-50.

64. Eva KW. The aging physician: changes in cognitive processing and their impact on medical practice. Acad Med. 2002;77:S1-6.

65. Kolb DA, Boyatzis RE, Mainemelis C. Experiential learning theory: Previous research and new directions. Perspect Thinking Learn Cogn Styles. 2001;1: 227-47
66. Lapkin S, Levett-Jones T. A cost-utility analysis of medium vs. high-fidelity human patient simulation manikins in nursing education. J Clin Nurs. 2011; 20:3543-52

67. Levin HM, McEwan PJ. Cost-effectiveness analysis: methods and applications. 2nd ed. Thousand Oaks: Sage; 2001.

68. Charlin B, van der Vleuten C. Standardized assessment of reasoning in contexts of uncertainty: the script concordance approach. Eval Health Prof. 2004;27:304-19.

69. Sheehan B, Kaufman D, Stetson P, et al. Cognitive Analysis of Decision Support for Antibiotic Prescribing at the Point of Ordering in a Neonatal Intensive Care Unit. AMIA Annu Symp Proc. 2009:2009:584-8.

\section{Submit your next manuscript to BioMed Central and we will help you at every step:}

- We accept pre-submission inquiries

- Our selector tool helps you to find the most relevant journal

- We provide round the clock customer support

- Convenient online submission

- Thorough peer review

- Inclusion in PubMed and all major indexing services

- Maximum visibility for your research

Submit your manuscript at www.biomedcentral.com/submit 